\title{
A novel handrail cueing system to prevent falls in older adults
}

\author{
Carol Y. Scovil PhD \\ Centre for Studies in Aging, Sunnybrook Health Sciences Centre \\ Philippe Corbeil PhD \\ Département de Médecine Sociale et Préventive, Université Laval \\ Tracy A. Lee MASc \\ Centre for Studies in Aging, Sunnybrook Health Sciences Centre \\ Sandra M. McKay PhD \\ Centre for Studies in Aging, Sunnybrook Health Sciences Centre \\ Amy L. Peters MHK \\ Centre for Studies in Aging, Sunnybrook Health Sciences Centre \\ Brian E. Maki PhD, PEng \\ Centre for Studies in Aging, Sunnybrook Health Sciences Centre; \\ Institute of Medical Science, Institute of Biomaterials and \\ Biomedical Engineering and Department of Surgery, \\ University of Toronto; Toronto Rehabilitation Institute \\ 2075 Bayview Avenue, Toronto, Ontario, M4N 3M5, Canada \\ E: brian.maki@sri.utoronto.ca
}

\begin{abstract}
C.Y. Scovil, P. Corbeil, T.A. Lee, S.M. McKay, A.L. Peters, Brian E. Maki. A novel handrail cueing system to prevent falls in older adults. Gerontechnology 2007; 6(4):224-229. Balance-recovery reactions that involve rapid reaching movements to grasp a handrail for support can play a critical role in preventing falls. To execute these reactions, the central nervous system (CNS) requires visuospatial information (VSI) about the handrail location. However, the urgent need to react rapidly may limit ability to acquire this information after loss of balance occurs. Instead, it appears that the CNS may continually monitor the environment and store salient VSI for use if and when a loss of balance occurs. Age-related deficits in visual attention may impair ability to use grasping reactions to recover balance by impeding the acquisition, processing and storage of required VSI. To counter this problem, we have developed a proximity-triggered handrail cueing system (patent pending) that provides a visual cue (flashing lights) and/or verbal cue ("attention, use the handrail") so as to attract attention to the handrail. The cueing system comprises light-emitting diodes mounted inside a translucent black railing, speakers inserted into the handrail mounting fixtures, and a photocell that triggers the cueing as the person approaches. To test the system, grasping reactions were evoked as subjects walked along an extended $(2 \times 6 \mathrm{~m})$ motion platform configured to simulate an office environment (including a stair, handrail and visual distracters). The platform was triggered to move suddenly and unexpectedly when the subject was adjacent to the handrail. Pilot results (21 subjects) showed that the rail was grasped more frequently when there was a verbal cue $(67 \%)$ or a visual cue $(40 \%)$, compared to conventional-handrail trials $(10 \%)$.
\end{abstract}

Keywords: Aging, attention, fall prevention, grasping, handrails, postural balance

Balance-recovery reactions that involve rapid reaching movements to touch or grasp an object for support can play a critical role in preventing falls, particularly in older adults who tend to be more dependent than younger persons in using 
the arms to respond to sudden loss of balance ${ }^{1}$. In order to reach to grasp or touch an object such as a handrail, the central nervous system (CNS) requires visuospatial information about the location of the target. However, for compensatory reaching reactions that are triggered by sudden unexpected or unpredictable loss of balance, the urgent need to react rapidly places severe temporal constraints on visuomotor processing. Recent results suggest that the CNS initiates these rapid perturbation-evoked movements using an internal egocentric 'visuospatial map' of the immediate surroundings that is formulated prior to perturbation onset and automatically updated on an ongoing basis as the person moves about ${ }^{2,3}$. This control strategy avoids the delay that would occur if it were necessary to construct a map to guide the compensatory movement after the onset of the balance perturbation. If and when a sudden unexpected loss of balance occurs, the pre-formed map can be used to immediately initiate a very rapid arm movement that is directed toward the nearest available handhold.

The need to monitor the environment suggests a critical role for the acquisition, processing and storage of visual information, involving various aspects of visual attention, gaze control and spatial working memory, all of which are known to decline with aging ${ }^{4-6}$. Although no studies have directly examined effects on control of balance reactions, there is evidence that common age-related visual-processing deficits can impair motor behaviour in situations that require visual monitoring of the surroundings. For example, age-related decline in the ability to rapidly extract information from the peripheral visual field predicts increased risk of driving accidents $^{7}$, and also correlates with reduced mobility ${ }^{8}$. In another study, older adults at high risk of falling were found to exhibit different gaze behaviour in comparison to low-risk subjects ${ }^{9}$, suggesting that the strategy used to gather visuospatial information during walking may affect risk of falling.
The present study examined the feasibility of countering adverse effects of age-related visual-processing deficits on balance control by using cueing to attract attention to salient features of the environment. In this initial study, we developed a novel handrail cueing system and performed pilot tests to examine whether the cueing increased the likelihood that subjects would look at the rail and grasp it in response to a sudden unexpected loss of balance.

\section{DeVelopMent OF THE SYSTEM}

The cueing system (patent pending) is designed to automatically (involuntarily) draw attention to a handrail as the person approaches. We propose that this 'attention capture' system will help to ensure that the handrail is incorporated into the individual's internal visuospatial map of the surroundings and thereby improve ability to rapidly and accurately reach to grasp the handrail for support if and when a sudden loss of balance occurs. In doing so, this device is intended to compensate for age-related deficits in visual attention that might otherwise have caused a failure to detect the presence of the handrail or to map its location accurately.

The visual-attention literature suggests that attention capture will be facilitated by locating the cues in close proximity to the rail, and by using cues that have a distinct onset $^{10}$. Attention capture may also be enhanced if the cue has symbolic features that are familiar and meaningful. There are certain generic symbols that most people tend to 'overlearn' in the course of their daily lives, and there is strong evidence that the appearance of an overlearned symbol in the visual field will produce an involuntary shift of attention ${ }^{11}$. Thus, for example, green or yellow flashing lights could draw attention to a handrail by taking advantage of overlearned associations with traffic lights and safety.

Based on these considerations, we have developed a handrail cueing system in which green or yellow light-emitting di- 
odes (LEDs), mounted internally along the longitudinal axis of a translucent railing, are triggered by a photoelectric proximitysensor to begin flashing as the individual approaches the handrail, thereby providing an abrupt onset cue (Figure 1). To enhance visibility and improve object recognition, the railing itself is black. This provides high contrast with respect to both the LEDs and typical backgrounds (for instance, white walls). High contrast with the background will also enhance ability to delineate the contours of the railing, which may facilitate motor planning of the finger and hand motion needed to grasp the rail ${ }^{12}$. A flashing frequency of $3 \mathrm{~Hz}$ was selected to promote attention capture ${ }^{13}$, while minimizing any potential danger to those at risk of photic-induced seizures ${ }^{14}$.

A second version of the cueing system involves a verbal prompt ("attention, use the handrail") that is triggered by the proximity sensor and delivered by speakers built into the rail or its mounting fixtures. Characteristics of the auditory cue were selected on the basis of ergonomic literature on the

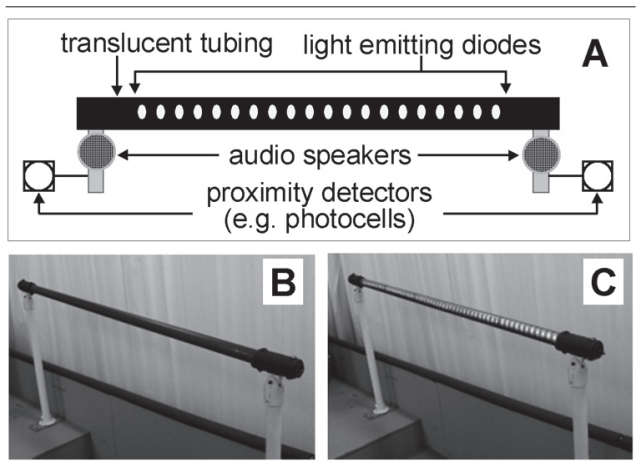

Figure 1. Schematic drawing (A) and photographs $(B, C)$ of the handrail cueing systems. During visual cueing, light-emitting diodes (LEDs) mounted within the translucent black railing are controlled to flash on $(C)$ and off $(B)$. Although yellow LEDs were used in the pilot tests described here, green LEDs are currently being used in our ongoing studies. Note that the speakers used during the auditory (verbal) cueing can be mounted on the handrail brackets (as depicted in A) or inserted within the ends of the handrail itself. The proximity sensors (photocells) that trigger the cueing are mounted on the walls near floor level, approximately $1.5 \mathrm{~m}$ from each end of the railing optimal design of warning systems: sound level $>15 \mathrm{~dB}$ above background noise ${ }^{15}$; speech (rather than abstract tone) ${ }^{16}$; female voice ${ }^{17}$; use of a signal word ("attention $\left.^{\prime \prime}\right)^{18}$; urgent tone ${ }^{19}$; length of the phrase (4-6 words) ${ }^{20}$; and number of repetitions (phrase is spoken twice) $^{20}$.

Like the visual cueing, the verbal prompt is primarily intended to enhance involuntary attention capture. In addition, however, the verbal prompt may influence voluntary behaviour by encouraging people to hold the rail before loss of balance can occur. In terms of maximizing safety, this is actually the most desirable outcome; however, we anticipate that this effect will not occur consistently. Rather, the influence of the cueing on the control of subsequent balance recovery reactions is expected to be a more consistent and robust benefit. By capturing attention, even momentarily, the cueing may help to incorporate the rail into the individual's visuospatial map and thereby facilitate rapid grasping of the rail in response to an unexpected loss of balance $^{21}$.

\section{Pilot testing \\ Methodology}

The handrail cueing systems were evaluated using an extended ( $2 \times 6 \mathrm{~m})$ motion platform configured to simulate a 'real-life' environment, including a stair, handrail and various visual distracters (Figure 2). The platform was triggered to move suddenly as the subject approached the handrail, and a deception was used to ensure that the perturbation was truly unexpected. To prevent learning and adaptation, subjects performed only one trial, which was their very first exposure to the perturbation and environment.

A door prevented viewing of the environment prior to the start of the trial. The subject was given the task of making a telephone call, which required opening the door, performing a visual search for the phone and walking to the far end of the platform to access the phone. The 


\section{Handrail cueing system}
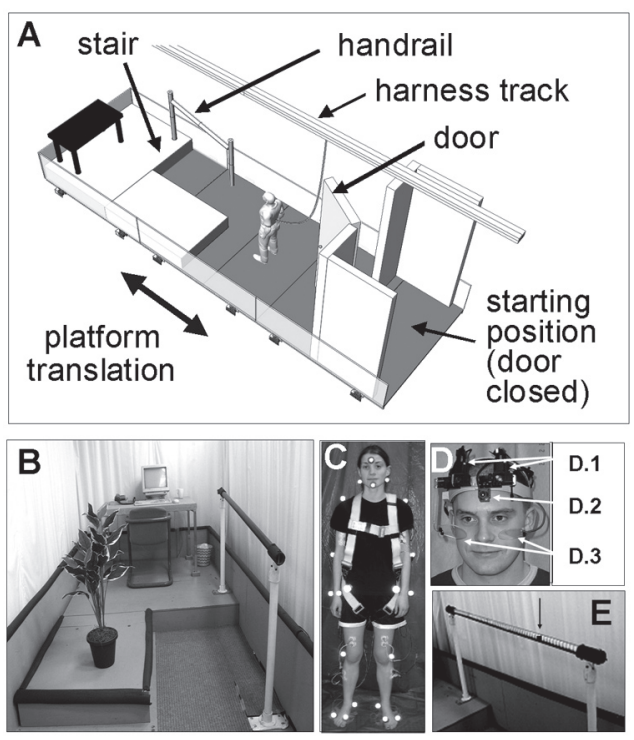

Figure 2. Experimental set-up used to study the effect of handrail cueing on gaze behaviour and reach-to-grasp reactions evoked by balance perturbation (platform motion) during ambulation: A. schematic drawing of the extended $(2 \times 6 \mathrm{~m})$ motion platform, configured to simulate a "real-life" environment; B. photograph of the view that the subject sees upon opening the door at the start of the trial; $C$. reflective markers placed on the body for purposes of motion analysis; $D$. head-mounted eye tracker used to determine the objects at which gaze was directed (includes: D. 1 infrared LEDS and cameras used to record corneal reflections; D.2 camera used to record the 'scene' that the head is facing, and D.3 semi-transparent lenses that reflect the infrared LED light to and from the eye); E. example output from the eye tracker, with the point-of-gaze location (the black dot indicated by the arrow) superimposed on the video recorded by the head-mounted scene camera (in the displayed video frame, the subject is looking at the handrail). In each trial, the subject is given a distraction task that requires walking to the end of the platform (for instance, make a phone call, using the phone located on the desk); in some trials, the platform is triggered by pressure switches to suddenly and unpredictably move forward or backward when the subject steps on the mat adjacent to the handrail

platform was triggered to move forward or backward suddenly and unexpectedly when the subject stepped on a pressure mat adjacent to the handrail, so as to evoke a reach-to-grasp reaction. The handrail cueing was triggered by a photocell when the subject was $1.4 \mathrm{~m}$ from the handrail, ensuring that the subject was exposed to the cueing for approximately $2 \mathrm{~s}$ before arriving at the handrail.

Three cueing conditions were tested during handrail development: (i) visual cue (flashing yellow LEDs), (ii) verbal cue ("attention, use the handrail"), and (iii) no cue (conventional handrail). 10 healthy, community-dwelling older adults (OA) aged 57-70 (4 male, 6 female) and 11 young adults (YA) aged 20-35 (7 male, 4 female) were assigned to one of these three conditions. Participants were required to have a corrected Snellen visual acuity of at least 20/40, and any volunteers with neurologic, orthopaedic or sensory disorders were excluded. Subjects provided written informed consent to comply with ethics approval granted by the institutional review board. In initial tests of the handrail cueing (10 subjects: 7 YA, 3 OA), subjects performed the 'telephone task' described above without balance perturbation (i.e., the platform did not move). Following these tests, 11 new subjects (4 YA, 7 OA) were tested using various perturbation magnitudes and directions (maximum platform acceleration, velocity and displacement of $3.5 \mathrm{~m} / \mathrm{s}^{2}, 1.0 \mathrm{~m} / \mathrm{s}$ and $0.43 \mathrm{~m}$, respectively; duration $0.6 \mathrm{~s})$. Based on previous studies ${ }^{1}$, it was expected that the selected perturbations would be sufficiently large to evoke a reach-to-grasp balance-recovery reaction in the majority of subjects.

A video-based motion-analysis system (Vicon-Peak, Oxford, UK) was used to record arm movements (Figure 2c), and a lightweight head-mounted eye tracker (Applied Science Laboratories, Bedford, MA, USA) was used to record eye movements and gaze direction (Figure $2 d$ ). The eye tracker superimposes the point-ofgaze on the video images recorded by a head-mounted 'scene' camera (Figure 2e), and thereby allows the object at which gaze was directed to be determined for each video frame (60 frames per second). For each trial, we determined whether the subject grasped the handrail and whether 
gaze was directed toward the handrail prior to grasping the rail.

\section{Results and discussion}

Results indicated that grasping of the rail (when walking for the first time in the unfamiliar environment) occurred more often when there was a verbal cue $167 \%$; 4 out of 6 subjects) or visual cue $(40 \%$; 2 out of 5 subjects), in comparison to a conventional rail with no cueing $(10 \%$; 1 out of 10 subjects). Gaze was measured for 18 (10 YA, $8 \mathrm{OA}$ ) of the subjects. Subjects were most likely to look at the rail when there was a verbal cue $(67 \% ; 4$ out of 6 subjects) or visual cue $(50 \% ; 2$ out of 4 subjects); however, a sizeable proportion of subjects also looked at the conventional rail $(38 \%$; 3 out of 8 subjects). Of the 5 subjects who grasped the rail (and had gaze data), only 2 looked directly at the rail before grasping it, suggesting that the other 3 were able to locate the rail using peripheral vision.

Although further testing is required to establish statistical significance, these pilot results do suggest that visual and/or verbal cueing may increase the tendency to grasp a handrail. Presumably, this occurs because the cueing attracts visual attention toward the rail; however, the extent to which this involves covert (peripheral vision) or overt (central vision) attention remains to be determined.

Comments from the pilot subjects raised some concerns regarding use of yellow cue lights. For example, the flashing yellow lights could be interpreted as a warning to avoid using the handrail or stairs. To evaluate such problems, 12 subjects were asked about their perceptions of the yellow cue lights, in comparison to green cue lights, after completing platform-perturbation trials with both colours. We selected LEDs with a wide viewing angle (90 degrees) and

\section{Acknowledgments}

Funded by the Canadian Institutes of Health Research and the Canadian Foundation for Innovation. The authors thank Dr. Jay Pratt for tested the brightest LEDs available in each colour $(5000 \mathrm{mcd}$ for green, $2500 \mathrm{mcd}$ for yellow). Most subjects (67\%) perceived the green lights as brighter, and more visible than the yellow lights (17\% perceived yellow as brighter, $17 \%$ perceived no difference). In addition, $50 \%$ of subjects preferred the green lights aesthetically $(25 \%$ preferred yellow, 25\% had no preference). 2 subjects interpreted the yellow lights as a warning not to touch the handrail, but no subjects interpreted the green lights in this way. On the basis of these results, we have elected to use green cueing lights in future studies. In addition, we plan to study the concurrent use of both visual and verbal cues, based on findings that congruent multi-modal stimuli are more effective in influencing behaviour of older adults, compared to stimuli that involve a single sensory modality ${ }^{22}$.

A full-scale study is now in progress to evaluate the responses of older adults (ages 64-80) to combined visual and verbal cues, as compared to visual cue alone (green LEDs) and no cueing (conventional handrail). Subjects are randomly assigned to one of the three handrail conditions, and their balance is perturbed as they approach the handrail while walking on the motion platform shown in Figure 2. This study will examine the timing, accuracy and other features of the reach-to-grasp reactions in detail, and will include analyses to assess involvement of peripheral versus central vision. We will also include analyses to verify that the cueing has no adverse effects, for instance, startle reactions or distracting effects that might actually increase risk of falling. If warranted by the results, future directions will include commercialization of the handrail cueing technology as a novel intervention to promote more effective handrail use and reduce risk of falling.

his expert advice, and gratefully acknowledge assistance provided by Adam Sobchek, James Tung, Aaron Marquis and Gerald Griggs in the construction of the motion platform and 
handrail cueing systems. This contribution has been presented at the $2^{\text {nd }}$ International Conference on Technology and Aging (Toronto, Canada; June, 2007).

\section{References}

1. Maki BE, Mcllroy WE. Change-in-support balance reactions in older persons: an emerging research area of clinical importance. Neurologic Clinics 2005;23(3):751-783

2. Ghafouri M, Mcllroy WE, Maki BE. Initiation of rapid reach-and-grasp balance reactions: is a pre-formed visuospatial map used in controlling the initial arm trajectory? Experimental Brain Research 2004;155(4):532-536

3. Zettel JL, Holbeche A, Mcllroy WE, Maki $B E$. Redirection of gaze and switching of attention during rapid stepping reactions evoked by unpredictable postural perturbation. Experimental Brain Research 2005;165(3):392-410

4. Salthouse TA. Reasoning and spatial abilities. In: Craik FIM, Salthouse TA, editors. The Handbook of Cognition and Aging. Hillsdale: Lawrence Erlbaum; 1992; pp 167-204

5. Munoz DP, Broughton JR, Goldring JE, Armstrong LT. Age-related performance of human subjects on saccadic eye movement tasks. Experimental Brain Research 1998;121(4):391-400

6. Kemps E, Newson R. Comparison of adult age differences in verbal and visuospatial memory: the importance of 'pure', parallel and validated measures. Journal of Clinical and Experimental Neuropsychology 2006;28(3):341-356

7. Owsley C, Ball K, McGwin G, Sloane ME, Roenker DL, White MF, Overley T. Visual processing impairment and risk of motor vehicle crash among older adults. Journal American Medical Association 1998;279(14):1083-1108

8. Owsley C, McGwin G. Association between visual attention and mobility in older adults. Journal of the American Geriatric Society 2004;52(11):1901-1906

9. Chapman GJ, Hollands MA. Evidence for a link between changes to gaze behaviour and risk of falling in older adults during locomotion. Gait and Posture 2006;24(3):288-294

10. Posner MI, Cohen YA. Components of visual orienting. In: Bouma $\mathrm{H}$, Bouwhuis DG, editors. Attention and performance X. Hillsdale: Lawrence Erlbaum; 1984; pp 531-556
11. Hommel B, Pratt J, Colzato L, Godijn R. Symbolic control of visual attention. Psychological Science 2000;12(5):360-365

12. Gentulucci M, Benuzzi F, Bertolani L, Gangitano M. Influence of stimulus color on the control of reaching-grasping movements. Experimental Brain Research 2001;137(1):36-44

13. Singh M, Kim S, Kim TS. Correlation between BOLD-fMRI and EEG signal changes in response to visual stimulus frequency in humans. Magnetic Resonance Medicine 2003;49(1):108-114

14. Harding G, Wilkins AJ, Erba G, Barkley GL, Fisher RS. Photic- and pattern-induced seizures: Expert consensus of the epilepsy foundation of America working group. Epilepsia 2005;46(9):1423-1425

15. Patterson RD. Auditory warning sounds in the work environment. Philosophical Transactions of the Royal Society of London. Series B, Biological Sciences 1990;327(1241):485-492

16. Edworthy J, Hellier E. Alarms and human behaviour: implications for medical alarms. British Journal of Anaesthesiology 2006;97(1):12-17

17. Edworthy J, Hellier E, Rivers J. The use of male or female voices in warning systems: a question of acoustics. Noise Health 2003;6(21):39-50

18. Edworthy J, Hellier E, Walters K, CliftMathews W, Crowther M. Acoustic, semantic and phonetic influence in spoken warning signal words. Applied Cognitive Psychology 2003;17(17):915-933

19. Hellier E, Edworthy J, Weedon B, Walters K, Adams A. The perceived urgency of speech warnings: semantics versus acoustics. Human Factors 2002;44(1):117

20. Han D, Zhou C, Liu Y, Zhai Y. [Ergonomical study on Chinese speech warning]. Space Medicine and Medical Engineering (Beijing) 1998;11(1):16-20

21. Maki BE, Cheng KC, Mansfield A, Scovil CY, Perry S, Peters AL, McKay SM, Lee TA, Marquis A, Corbeil P, Fernie GR, Liu B, Mcllroy WE. Preventing falls in older adults: New interventions to promote more effective change-insupport balance reactions. Journal of Electromyography and Kinesiology (in press; E-publication ahead of print, DOI 10.1016/j.jelekin.2007.06.005).

22. Laurienti PJ, Burdette JH, Maldjian JA, Wallace MT. Enhanced multisensory integration in older adults. Neurobiology of Aging 2006;27(8):1155-1163 